\title{
Junge Alte als neue „Urbaniten“? Mobilitätstrends der Generation 50plus
}

\author{
Caroline Kramer $\cdot$ Carmella Pfaffenbach
}

Eingegangen: 31. Mai 2010 / Angenommen: 7. Januar 2011 / Online publiziert: 5. Februar 2011

(C) Springer-Verlag 2011

Zusammenfassung In der aktuellen wissenschaftlichen Diskussion zur Wohnmobilität von Älteren werden zwei konträre Prozesse behandelt: Einerseits wird davon ausgegangen, dass sich zukünftige Ältere bezüglich ihrer Lebenskonzepte und deren Lokalisierung analog zu den jetzigen Älteren verhalten werden und in einem nennenswerten Umfang im Ruhestand die Kernstädte verlassen, um sich im suburbanen Raum niederzulassen. Andererseits wird eine fundamentale Trendwende im Sinne einer Reurbanisierung und eine Renaissance der Städte für alle Altersgruppen und damit auch für die Generation 50plus erwartet. Für eine Untersuchung in drei deutschen Großstadtregionen, deren Ergebnisse in diesem Beitrag diskutiert werden, wurde zunächst prinzipiell davon ausgegangen, dass sich die Generation 50plus als Seniorengeneration von der heute älteren Generation deutlich unterscheiden wird, andere Lebensstile ausprägt, die vermutlich auch an anderen (Wohn-)Standorten realisiert werden. Die Auswertung der empirischen Daten ergab, dass die Befragten in den Großstadtregionen weder in größerem Ausmaß planen, die Stadt im Alter zu verlassen, noch zieht es die ehemaligen „Suburbaniten“ in großer Zahl in die Städte. Für die Zeit im Ruhestand ist somit eine Persistenz bezüglich des Hauptwohnsitzes geplant. Wenn man eine Renaissance der Städte jedoch daran festmacht, dass Städte heute weniger oft als früher ver-

Prof. Dr. C. Kramer $(\square)$

Institut für Geographie und Geoökologie, Karlsruher Institut

für Technologie (KIT), 76131 Karlsruhe,

Reinhard-Baumeister-Platz 1, Deutschland

E-Mail: caroline.kramer@kit.edu

Prof. Dr. C. Pfaffenbach

Geographisches Institut, RWTH Aachen University,

Templergraben 55, 52056 Aachen, Deutschland

E-Mail: pfaffenbach@geo.rwth-aachen.de lassen werden, dann sind auch für die Generation 50plus Reurbanisierungstendenzen feststellbar.

Schlüsselwörter Reurbanisierung · Suburbanisierung · Migrationsabsichten · Generation 50plus ·

Großstadtregionen

\section{The Young Elderly as New "Urbanites"? The Generation 50plus and Its Mobility Trends}

Abstract Senior citizens in general have different expectations regarding their life concepts and the spatial localization of such following their retirement. The ongoing scientific research on the residential mobility of the elderly discerns two contrary processes. Some presume the future senior citizens will act in an analogous way to the elderly nowadays. Thus upon retiring they will leave the city to relocate to the suburbs. Others foresee a trend reversal towards a re-urbanisation accompanied by the renaissance of the city for all age groups including the generation 50plus. These developments were the focus of a study conducted in three major metropolitan areas; the results are discussed in this article. The study assumed that as the generation 50plus grows older their lifestyles and places of residence among other things will differ from the senior citizens of today. The evaluation of the empirical data showed, that the respondents in the greater metropolitan areas did not intend to move to the suburbs upon retirement. Neither do the respondents in the suburbs plan to move to the city. In other words a tendency towards remaining in the current residential areas was detected for the majority of the respondents. Thus if one defines the renaissance of the city by the decreasing number of people leaving the cities, then 
we can also identify tendencies towards re-urbanisation in the generation 50plus.

Keywords Re-urbanisation - Suburbanisation - Intentions to migrate $\cdot$ Generation 50plus $\cdot$ Metropolitan areas

\section{Wohnpräferenzen der Generation 50plus vor dem Hintergrund des demographischen Wandels: Fragestellung und Herangehensweise}

\subsection{Einleitung und Fragestellung}

Über die Lebensstile und Wohnansprüche der künftigen älteren Generation ist bislang wenig bekannt. Die bisherigen Arbeiten zum demographischen Wandel in Deutschland, auch in einzelnen Großstädten, konzentrieren sich vor allem auf die quantitative Veränderung der Kohorten. In der aktuellen wissenschaftlichen Diskussion zur Wohnmobilität von Älteren werden zwei konträre Prozesse behandelt: Entweder wird davon ausgegangen, dass sich zukünftige Ältere bezüglich ihrer Lebenskonzepte und deren Lokalisierung analog zu den jetzigen Älteren verhalten werden und in einem nennenswerten Umfang im Ruhestand die Kernstädte verlassen, um sich im suburbanen Raum niederzulassen (z. B. Hirschle/Schürt 2008: 217; weitere Diskussion in Kap. 2.1), oder es wird eine fundamentale Trendwende im Sinne einer Reurbanisierung und eine Renaissance der Städte für alle Altersgruppen und damit auch für die Generation 50plus erwartet (z. B. Brühl/Echter/Frölich von Bodelschwingh et al. 2005; weitere Diskussion in Kap. 2.2).

Wir gehen davon aus, dass sich die Generation 50plus (in unserer Studie die Jahrgänge 1945 bis 1954) als Seniorengeneration von der heute älteren Generation (der heute über 65-Jährigen) deutlich unterscheiden wird, da sie durch Bildungsexpansion, Emanzipation und Partizipation über andere Ansprüche und Potenziale verfügt. Es handelt sich um die erste Nachkriegsgeneration, die als junge Erwachsene in der Phase der gesellschaftlichen Umbrüche und eines generellen Wertewandels Ende der 1960er Jahre zahlreiche Veränderungen initiiert und miterlebt hat. Sie zählen zu der Gruppe, in der Prozesse wie die Individualisierung ihren Ausgang genommen haben, und sie haben von der frühen Bildungsexpansion profitiert (vgl. u. a. Beck 1986: 127; Inglehart 1998: 85 ff.). Dies hat vor allem auch auf

\footnotetext{
${ }^{1}$ Diese Jahrgänge sind zum einen mit dem Startjahr unserer ersten empirischen Untersuchung zu begründen: im Jahr 2005 begann das Pilotprojekt in München mit den damals 51-60-Jährigen (Geburtskohorte 1945-1954). Im weiteren Verlauf wurde u. a. aus Gründen der Vergleichbarkeit diese Alterskohorte beibehalten. Zum anderen wurden dadurch auch bei den später durchgeführten Untersuchungen in Aachen und Karlsruhe ausschließlich Personen erfasst, die noch nicht im gesetzlichen Rentenalter sind.
}

die Frauen dieser Altersgruppe insofern nachhaltige Wirkung, als diese Gruppe nicht nur deutlich besser ausgebildet wurde als ihre Mütter, sondern auch aufgrund eigener Erwerbstätigkeit im nun bevorstehenden Rentenalter über andere finanzielle Ressourcen verfügt als die heutigen Rentnerinnen. Im Gegensatz dazu hat die heute ältere Generation den größten Teil ihrer Jugend in den noch wesentlich konservativeren Kriegs- und Nachkriegsjahren verbracht, und Frauen absolvierten seltener eine Berufsausbildung oder gar ein Studium. Diese Generation kann allerdings heute meist über einen gewissen Wohlstand im Alter verfügen, was auf die künftige Rentnergeneration nicht mehr in diesem Maße zutreffen dürfte. Sie wurde aufgrund von tief greifenden Veränderungen des Arbeitsmarktes mehr als die vorherige Generation von befristeten Beschäftigungsverhältnissen, Arbeitslosigkeit, zunehmender (Schein-)Selbstständigkeit etc. betroffen, was zu Brüchen in den Erwerbsbiographien führte und zudem Auswirkungen auf die Höhe und Sicherheit der zu erwartenden Renten hat.

Sie werden deshalb andere Senioren sein als die bisherigen und andere Lebensstile ausprägen (vgl. Kramer/Pfaffenbach 2007), die vermutlich auch an anderen (Wohn-)Standorten realisiert werden. Aus diesem Grund geht der vorliegende Beitrag folgender Fragestellung nach: Wie sehen die Lebenskonzepte der Generation 50plus für ihren Ruhestand aus und wo und wie sollen diese realisiert werden?

Unter Lebenskonzepten wird hier die Planung für den bevorstehenden Ruhestand verstanden. Lebenskonzepte umfassen sowohl Planungen des künftigen Wohnstandortes, der Wohnform und des künftigen Lebensstils als auch Haltungen gegenüber dem Älterwerden und der Altersvorsorge. Die Lebenskonzepte werden von individuellen Merkmalen (beispielsweise Geschlecht, Bildung, sozialer Status) geprägt, die nicht selten als constraints auf die Realisierbarkeit der Lebenskonzepte einwirken. Alle Planungen des künftigen Wohnens stehen zudem in engem Zusammenhang mit dem aktuellen räumlichen Kontext (Wohnsituation, Wohnumfeld etc.) und der bisherigen Wohnbiographie, da darauf aufbauend für die nähere Zukunft geplant wird, z. B. hinsichtlich der Frage, ob ein Verbleiben nach der Verrentung gewünscht oder noch finanzierbar ist. In diesem Beitrag werden insbesondere Lebenskonzepte der Generation 50plus zum künftigen Wohnen thematisiert.

Bedeutsam ist diese Frage aus einer stadt- und sozialgeographischen Perspektive insbesondere, weil die Wohnstandortwahl der künftigen Senioren Auswirkungen auf die Entwicklung der Raumstrukturen (z. B. Wohnungsmarkt, Infrastruktur) vermuten lässt. Der aktuellen Diskussion der stadt- und sozialgeographischen Forschung zufolge müssten abnehmende Suburbanisierungstendenzen und zunehmende Reurbanisierungstendenzen zu beobachten sein (vgl. Kap. 2.1 und 2.2). Die Ansprüche dieser Altersgruppe an die strukturellen Rahmenbedingungen ihrer urbanen oder sub- 
urbanen Wohnorte werden eine große Herausforderung darstellen, da es sich um geburtenstarke Jahrgänge handelt. Die strukturellen Rahmenbedingungen betreffen in erster Linie die bauliche Ausgestaltung der Wohnung, z. B. hinsichtlich der Größe und Barrierefreiheit, als auch die Verkehrserschließung sowie die Zugänglichkeit der Infrastruktur des Wohnumfeldes.

\subsection{Theoretischer Hintergrund}

Aus einer theoretisch-konzeptionellen Perspektive stehen handelnde Menschen im Vordergrund. Für die Konstitution des Handelns sind verschiedene Komponenten bedeutsam. Werlen (2007: 593) versteht unter diesen Komponenten sowohl sozial-kulturelle und subjektive als auch physisch-materielle. Sie können als constraints im Sinne von Hägerstrand (1975) und als Strukturen im Sinne der Strukturationstheorie von Giddens (1997) interpretiert werden. So sind nicht allein subjektive Komponenten für das Handeln entscheidend, wie dies von der Strukturationstheorie postuliert wird, die insbesondere auf so genannte Mikro-Strukturen fokussiert ist, sondern die Entscheidungsfreiheit des Individuums wird auch durch den jeweiligen zeitlichen, räumlichen und sozialen Kontext bedingt (vgl. Meusburger 1999: 96 ff.). Letzteres ist die Sichtweise des constraints-Ansatzes, wobei eher MakroStrukturen eine zentrale Rolle spielen.

Die Hägerstrand'schen constraints existieren nicht an sich, sondern werden durch Handeln reproduziert. Zum einen gestalten die Akteure ihren Handlungskontext, zum anderen stellt dieser Kontext Bedingung oder constraint ihres Lebensalltags dar. Somit ist das Verständnis von Kontext und constraints hier nicht deterministisch, sondern probabilistisch und im Sinne von Weichhart (2003: 34 ff.; 2008: 323 f.) als ,action setting“ zu verstehen.

Auch Giddens geht von einer wechselseitigen Beziehung aus, derjenigen zwischen Handlung und Struktur, und bezeichnet sie als Dualität (Giddens 1997: 77). Strukturen sind handlungsrelevant, weil sie Handlungen und Handlungsspielräume beeinflussen können. Strukturen schränken die Handelnden aber nicht nur ein, sondern ermöglichen auch Handlungen, indem sie Handlungsorientierungen vorgeben. Allerdings existieren Strukturen nicht aus sich heraus, sondern werden von den Handelnden geschaffen, d. h. produziert und reproduziert. Strukturen sind damit einerseits das Ergebnis vergangener Handlungen und stellen andererseits Bedingungen für aktuelles und zukünftiges Handeln dar (Giddens 1988: 290; Giddens 1997: 52). Strukturen werden von Giddens als Ressourcen und Regeln definiert. In der vorliegenden Studie sind im Hinblick auf die Wohnstandortwahl für den Ruhestand und Migrationsabsichten insbesondere die finanzielle Situation der Akteure (als Ressource und Mikro-Struktur) und Wohnungsmarktstrukturen (als Makro-Struktur) relevant.
In der sozialwissenschaftlichen Alternsforschung bilden soziale Lage (z. B. verwitwete Rentnerin) und Lebenslage (Höhe des Renteneinkommens, Bildungsniveau) die äußere Situation des Handelns alter Menschen. Diese äußeren Einflüsse werden jedoch erst durch eine subjektive Wahrnehmung und Interpretation handlungsrelevant (Amrhein 2004: 68). Neben der finanziellen Situation (ökonomisches Kapital) und dem Bildungsniveau (kulturelles Kapital) sind auch das Netz von Freunden und Verwandten (soziales Kapital) sowie die körperliche und geistige Gesundheit (körperliches Kapital) bedeutsam. Amrhein (2004: 80) plädiert bei der Analyse der Lebensrealität älterer Menschen zudem für die Integration einer Lebenslauf- und Biographieperspektive, da Handlungsmuster und Lebensstile im Alter auch ein Produkt der individuellen Biographie und der Geschichte der Kohorte sind (zur geographischen Biographieforschung vgl. auch Pfaffenbach 2005).

\subsection{Methodisches Vorgehen}

Eine größtmögliche Vielfalt an Lebenskonzepten ist im großstädtischen Kontext zu erwarten, wo sich die meisten Möglichkeiten zur Realisierung verschiedener Lebenskonzepte finden lassen. Aus diesem Grund konzentrierten sich unsere Untersuchungen zunächst auf die Großstadtregion München. Aufgrund der Zuwanderung ehemals junger Arbeitsbevölkerung in den 1970er und 1980er Jahren ist in der Landeshauptstadt Bayerns eine besonders große Kohorte dieser zukünftig , anderen' Älteren anzutreffen, so dass neue Entwicklungen in dieser Generation hier in stärkerem Ausmaß erkennbar sind als in anderen Städten Deutschlands. ${ }^{2}$ Im Anschluss an die Münchner Pilotstudie wurde die Untersuchung inzwischen auch in Aachen und Karlsruhe durchgeführt. ${ }^{3}$ Auch diese beiden Hochschulstandorte zählen zu den eher prosperierenden Großstädten mit hohem Potenzial an gut Gebildeten und geringen Abwanderungsraten der Generation 50plus. Es sind jedoch aufgrund anderer Wohnungsmarktstrukturen andere Zukunftspläne bezüglich der Wohnstandorte zu erwarten als in München.

In München wurden fünf Stadtbezirksteile für die Untersuchung ausgewählt. Die Stadtbezirksteile sollten unterschiedlich strukturiert sein, damit eine möglichst große Vielfalt unterschiedlicher "Schauplätze des Handelns“ berücksichtigt und analysiert werden kann. Als strukturelle

\footnotetext{
${ }^{2}$ Die Durchführung der Pilotstudie in München wurde ebenso wie die geplanten Erhebungen in Berlin, Bochum, Leipzig, Kaiserslautern, Mannheim und Schwerin dankenswerterweise von der Deutschen Forschungsgemeinschaft gefördert.

${ }^{3}$ Die Untersuchungen in Aachen und Karlsruhe erfolgten in Lehrprojekten. Den beteiligten Mitarbeiterinnen und studentischen Hilfskräften Maike Didero, Melanie Kappler, Marta Kowalski, Agata Siuda und Nicola Sulke sowie den teilnehmenden Studierenden sei an dieser Stelle herzlich für ihr Engagement gedankt.
} 
Merkmale wurden u. a. das Durchschnittsalter der Bewohner, die durchschnittliche Wohnungs- und Haushaltsgröße, der Frauenanteil bei Akademikern, das Alter der Wohnbebauung sowie die Lage im Stadtgebiet verwendet. Die Bandbreite der Stadtbezirksteile reicht von einem alternativen Szene- und Single-Viertel (Isarvorstadt) über Viertel der gehobenen Mittelschicht (Schwabing-West, Alt-Bogenhausen), einem typischen Arbeiterviertel (Obergiesing) bis zu einem Einfamilienhausviertel mit überdurchschnittlich großen Haushalten (Allach/Untermenzing-West). Zugleich sollten die Stadtbezirksteile hinsichtlich der sozialen und räumlichen, vor allem der baulichen Struktur in sich möglichst homogen sein. In Aachen und Karlsruhe erfolgten die Untersuchungen im gesamten Stadtgebiet. Kleinräumliche Differenzierungen auf der Ebene von Stadtbezirken wurden hier aufgrund der geringeren Einwohnerzahl der beiden Städte (260.000 bzw. 290.000 Einwohner) erst bei der Auswertung relevant.

Da nicht nur urbane, sondern auch suburbane Lebenskonzepte erfasst werden sollten, wurde die Untersuchung auf jeweils zwei Umlandgemeinden in jeder Stadtregion ausgedehnt. Die Stadt Dachau und die Gemeinde Vaterstetten im Münchner Umland unterscheiden sich strukturell insofern voneinander, als Dachau als Mittelstadt über ausgeprägte städtische Strukturen mit umfangreichen Angeboten und eine dennoch überschaubare Lebenswelt (vor allem im Unterschied zu München) verfügt, während Vaterstetten eher als Schlafstadt bezeichnet werden kann, deren Bewohner in ihrer Aktivität vielfach stärker auf die Großstadt München ausgerichtet sind. Für die Auswahl der Umlandgemeinden wurden sowohl die Merkmale der zentralörtlichen Funktionen als auch die Pendlerverflechtungen berücksichtigt. Diese beiden Gemeinden stehen stellvertretend für zwei häufiger anzutreffende Gemeindetypen im suburbanen Raum Münchens.

Die Untersuchungen im suburbanen Umland von Aachen erfolgten in den Städten Herzogenrath und Würselen, die beide im Norden der Kernstadt Aachen liegen und enge Verflechtungen mit ihr aufweisen. Andere Gemeinden im suburbanen Umland Aachens befinden sich entweder aufgrund der Grenznähe bereits im Ausland (z. B. Vaals in den Niederlanden und Kelmis in Belgien) oder sie wurden eingemeindet (z. B. Kornelimünster). Die Stadt Würselen verfügt als Mittelstadt mit einem lebendigen Gewerbegebiet über zentralörtliche Funktionen, die sogar einen leichten Einpendlerüberschuss bewirken. Die Stadt Herzogenrath kann dagegen als klassische suburbane Gemeinde charakterisiert werden, die derzeit jedoch rückläufige Wachstumserwartungen aufweist.

Die Untersuchungen im suburbanen Umland von Karlsruhe wurden in Weingarten/Baden und LinkenheimHochstetten durchgeführt, die beide im engeren nördlichen Verflechtungsbereich Karlsruhes liegen und sich wie die
Gemeinden im Münchner und Aachener Umland deutlich strukturell voneinander unterscheiden. Die Gemeinde Weingarten zeichnet sich durch einen historischen Ortskern mit einer gewissen Eigenständigkeit hinsichtlich des Einkaufsund Freizeitangebotes aus. Die Gemeinde LinkenheimHochstetten besteht in dieser Form erst seit 1974 und kann eher als Schlafstadt bezeichnet werden. Sie besitzt jedoch eine sehr gute Verkehrsanbindung mit dem ÖPNV an die Karlsruher Innenstadt.

Das empirisch-methodische Vorgehen wurde als Kombination qualitativer und quantitativer Methoden konzipiert. Den Befragungen lagen Zufallsstichproben von Einwohneradressen der entsprechenden Altersgruppe aus den Melderegistern zugrunde. In der Münchner Pilotstudie bildeten qualitative Interviews die erste Phase. Inhaltlich ging es um die Lebenskonzepte, die Altersstereotype und die München-Bilder der Untersuchungsgruppe. ${ }^{4}$ Für die Leitfadeninterviews wurden zufällig ausgewählte Personen der Stichprobe telefonisch kontaktiert und es wurde mit ihnen ein Interviewtermin vereinbart. Pro Stadtteil und Umlandgemeinde wurden fünf Interviews durchgeführt, die meist rund eine Stunde Interviewzeit umfassten.

Die standardisierte Befragung zielte auf die Erfassung der aktuellen Wohnsituation, ihre Bewertung und die Planung für die nähere Zukunft, auf die Erfassung der derzeitigen und geplanten Freizeit- und Urlaubsgestaltung, der beruflichen, finanziellen und gesundheitlichen Situation sowie die Einstellungen zum Älterwerden. Der Rücklauf lag in allen Gemeinden bei rund einem Drittel. ${ }^{5}$ Die Verteilung der Befragten über die Geschlechter zeigt in allen Städten etwas höhere Rückläufe der Frauen, und es sind - wie bei allen Befragungen - Personen mit Hochschulreife leicht über- und Personen mit niedrigem oder keinem Schulabschluss leicht unterrepräsentiert. Allerdings verteilen sich die Bildungsabschlüsse über die Stichproben dennoch recht gleichmäßig: Je ein Drittel aller Befragten hat eine Hochschulreife, einen Realschulabschluss oder einen Hauptschulabschluss (bzw. vergleichbare Abschlüsse).

\footnotetext{
${ }^{4}$ Entsprechende qualitative Untersuchungen in Aachen und Karlsruhe werden folgen.

${ }^{5}$ Der Rücklauf lag in München bei $25 \%$, in Dachau bei $44 \%$ und in Vaterstetten bei $54 \%$, so dass insgesamt $34 \%$ der angeschriebenen Personen den Fragebogen ausgefüllt zurückgeschickt haben (Gesamtzahl der auswertbaren Fragebögen: 543). In Aachen beteiligten sich $33 \%$, in Herzogenrath $32 \%$ und in Würselen $27 \%$ der angeschriebenen Personen ( $32 \%$ im Gesamtdurchschnitt; 925 auswertbare Fragebögen). In Karlsruhe, Weingarten und Linkenheim-Hochstetten lag der Rücklauf bei je $35 \%$ (35 \% im Gesamtdurchschnitt, 1.112 auswertbare Fragebögen).
} 


\section{Wohnpräferenzen der Generation 50plus für die Zukunft}

Die räumliche Mobilität ist altersspezifisch sehr unterschiedlich (Wagner 1989; Bucher/Heins 2001). Sie erreicht in der Zeit zwischen dem 20. und dem 30. Lebensjahr ihren Höhepunkt, sinkt dann stark ab und steigt ab dem 65 . bzw. dem 75. Lebensjahr wieder leicht an. Jüngere Studien (Eichener 2001; Heinze/Eichener/Naegele et al. 1997) schätzen auf der Basis des sozioökonomischen Panels (SOEP) sogar einen erheblichen Anstieg der Mobilität insbesondere von Mieterhaushalten im Laufe des dritten Lebensabschnitts. Dagegen hat Friedrich (2009) seit Mitte der 1990er Jahre einen Rückgang der Binnenwanderungsraten der 65-Jährigen und Älteren feststellen können.

Aus der Migrationsforschung ist bekannt, dass Ereignisse im Lebenslauf, wie Heirat, Geburt von Kindern oder Auszug der Kinder, Anlässe für Mobilität sind (Bucher/Heins 2001: 120 ff.; Flöthmann 1997: 25). Bei der Analyse von Migrationsprozessen ist eine regional differenzierte Betrachtungsweise aus zahlreichen Gründen notwendig. So gestalten sich die Handlungsoptionen in einem entspannten Wohnungsmarkt nach dem Auszug der Kinder oder dem Tod des Partners anders als in einem bedrängten und hochpreisigen Wohnungsmarkt, wie z. B. in München. In biographischen Wanderungsstudien konnte zudem nachgewiesen werden, dass Wohnortwechsel in Städten insgesamt enger mit Veränderungen in der familialen Biographie als mit der Erwerbsbiographie verbunden sind (Flöthmann 1997: 43 f.). Für die Generation 50plus könnte dies bedeuten, dass der Auszug der Kinder, die Trennung vom Partner, der Tod des Partners, das Eingehen einer neuen Partnerschaft oder andere Veränderungen im Privatleben häufiger einen Umzugsgrund darstellen als der Eintritt in den Ruhestand. Betrachtet man die zentralen Wanderungsmuster und -motive älterer Menschen, so zeigt sich, dass sie zu rund zwei Drittel nur innerhalb einer Distanz von $50 \mathrm{~km}$ umziehen und dass die Migrationen häufig auch dann auf private Netzwerke von Angehörigen ausgerichtet sind, wenn nicht direkt zu Familienangehörigen gezogen wird (Friedrich 2001a: 125).

Im Folgenden werden wir drei Trends der Wohnstandortpräferenzen der Generation 50plus diskutieren: Suburbanisierung, Reurbanisierung und Persistenz am jetzigen Wohnstandort. Ein weiterer Mobilitätstrend, der für die Altersgruppen der jetzigen und künftigen Senioren derzeit zunimmt und auch zunehmend diskutiert wird, der Umzug in den „sonnigen Süden“ (vgl. z. B. Friedrich/Kaiser 2001; Breuer 2003; Breuer 2004; Breuer 2005; Breuer/Müller 2005; Buck 2005), wird in dem vorliegenden Beitrag ausgeklammert, da er für Aachen und Karlsruhe nur eine marginale Bedeutung aufweist und auch in München eher selten dauerhafte Wohnsitzwechsel angestrebt werden (vgl. Kramer/Pfaffenbach 2009: 166 f.). Daher ist anzunehmen, dass von Altersruhesitzwanderungen ins Ausland kaum nennenswerte Auswirkungen auf die Raumstrukturen der deutschen Großstadtregionen ausgehen werden.

Bei der Diskussion der drei Trends werden wir zunächst auf aktuelle Literatur und Daten zurückgreifen und diese anschließend den Ergebnissen unserer Untersuchungen in München, Aachen und Karlsruhe gegenüberstellen. In dem zugrundeliegenden Fragebogen wurde in einem ersten Schritt erfasst, ob man sich vorstellen könne, mit Beginn des Ruhestandes bzw. dem des Partners die Wohnsituation zu verändern. In einem zweiten Schritt wurde dann nach bereits bestehenden Umzugsplänen und deren Zielen gefragt. Den konkreten Planungen wird in diesem Zusammenhang die größere Relevanz beigemessen, da bloße Vorstellungen eines Wohnortwechsels als zu vage angesehen werden. Zudem ist diesen Angaben zu konkreten Planungen - trotz der Prospektivität - eine große Glaubwürdigkeit beizumessen (vgl. Boerner/Schramm 1998: 88).

\subsection{Raus aus der Stadt: Umzug in den suburbanen Raum}

Suburbanisierung als Wohnstandortpräferenz wird in der Regel mit bestimmten soziodemographischen Strukturen verknüpft. Es sind in den 1960er, 1970er und 1980er Jahren insbesondere gut verdienende junge Familien mit Kindern gewesen, die die Kernstädte verlassen haben und in Umlandgemeinden zumeist ins Wohneigentum gezogen sind. Erst zu einem späteren Zeitpunkt sind vermehrt auch Haushalte zu Trägern des Prozesses geworden, die von diesem klassischen Muster abweichen. In den ostdeutschen Großstadtregionen zogen z. B. Haushalte aller Altersstufen und Größen in das suburbane Umland, wo sie häufig auch zur Miete wohnen, was mittlerweile auch im Westen zu beobachten ist (Herfert/Schulz 2002: 124). Ältere Generationen nehmen offenbar seit den 1980er Jahren stärker an Suburbanisierungsprozessen teil als in den Jahrzehnten zuvor.

Bei einer bundesweiten Betrachtung der Altersselektivität von Wanderungsprozessen fällt auf, dass die deutschen Kernstädte aktuell insgesamt eine negative Wanderungsbilanz der 65-Jährigen und Älteren aufweisen, wohingegen die Umlandkreise zu den Gewinnern dieser Altersgruppe zählen (vgl. auch Bucher/Heins 2001: 123 f.; Hirschle/Schürt 2008: 217). Dieses Muster kann für zahlreiche Großstadtregionen nachgewiesen werden, insbesondere für Frankfurt am Main, Hamburg, Stuttgart und München, d. h. für dynamische Großstadtregionen mit angespanntem Wohnungsmarkt. Die bisher beobachteten Abwanderungsraten der Älteren aus den Städten ließen sogar schon Befürchtungen laut werden, dass die bestehende altengerechte, soziale Infrastruktur in 


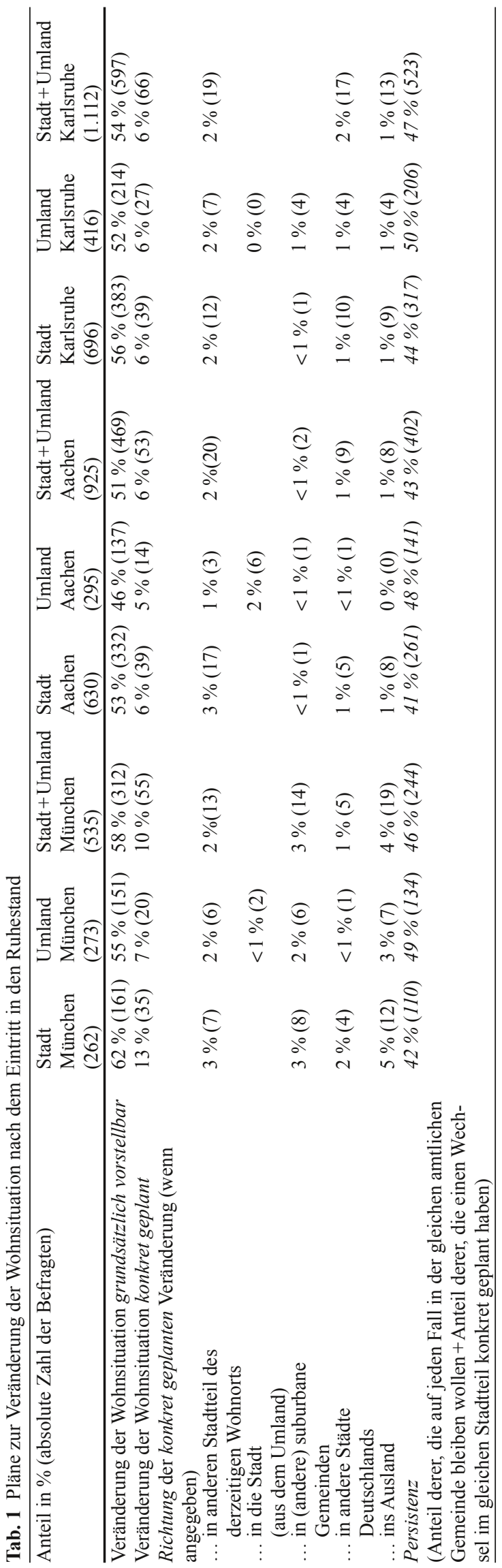

den Städten in Zukunft nicht ausgelastet sein könne (Friedrich 2001a: 125). ${ }^{6}$

Sowohl für die Altersgruppe der 50- bis 65-Jährigen als auch der über 65-Jährigen führt München die Liste der deutschen Großstädte mit den höchsten Wanderungsverlusten an, ${ }^{7}$ wobei als Wanderungsmotiv am häufigsten ein angemessenes Preis-Leistungs-Verhältnis beim Wohnungs- bzw. Hauskauf angegeben wurde (Referat für Stadtplanung und Bauordnung der Landeshauptstadt München 2002). Gerade in der Gruppe der über 75-Jährigen weist München hohe Wanderungsverluste auf, die überwiegend aus ökonomischen Gründen, aus einem Mangel an bezahlbaren Altenheim- und Pflegeplätzen erfolgen (Referat für Stadtplanung und Bauordnung der Landeshauptstadt München 2004).

Auch in Karlsruhe und Aachen sind leichte Wanderungsverluste der über 55-Jährigen zu beobachten, die vorwiegend von den Außenstadtbereichen in das unmittelbar benachbarte suburbane Umland gerichtet sind. Als zentrale Motive werden die Eigentumsbildung und eine „Ruhesitzorientierung“ genannt (Stadt Karlsruhe 2009: 28 und 72; Wörmer 2010: 66). In vielen der in München durchgeführten qualitativen Interviews wurde geäußert, im Ruhestand eine kleinere, vor allem aber eine billigere Wohnung suchen zu wollen, die vorzugsweise im selben Viertel oder am Stadtrand, jedoch weniger im suburbanen Raum liegen sollte.

Für die Analyse der Suburbanisierungstendenzen im Rahmen dieser Studie wurde nur von denjenigen Befragten ausgegangen, die angaben, bereits konkrete Pläne für einen Wohnortwechsel im Ruhestand zu haben (München $\mathrm{n}=35$ (13\%), Aachen $n=39$ (6\%), Karlsruhe $n=39$ (6\%); vgl. Tab. 1). Einen Umzug in eine suburbane Gemeinde Münchens oder in eine andere Stadt in Deutschland haben $5 \%$ der Münchner Befragten bereits konkret geplant (3\% ins suburbane Umland, $2 \%$ in eine andere deutsche Stadt), wobei als Umzugsmotiv neben dem ländlichen Umfeld auch das kostengünstigere Wohnen ein wichtige Rolle spielt. In Aachen und Karlsruhe planen nur 1-2 \% der Befragten einen Umzug in das Umland oder eine andere deutsche Stadt. Somit sind nur in der Region München potenzielle künftige „Sub-“ und „Exurbaniten“ zu erwarten. In den anderen beiden Städten planen nur sehr wenige Befragte einen Wechsel in das suburbane Umland, nicht zuletzt, da sie weitaus häufiger bereits in der Stadt Wohneigentum erwerben konnten und das Preisniveau des städtischen Wohnungsmarktes deutlich niedriger ist als in München. Es ist somit festzuhalten, dass die Suburbanisierungstendenzen bei den hier Befragten

\footnotetext{
${ }^{6}$ So übersteigt laut Aussage der Leiterin des Seniorenbüros der Stadt Karlsruhe, Kerstin Safian, das Angebot der Alten- und Pflegeheimplätze seit einiger Zeit die Nachfrage in Karlsruhe.

${ }^{7}$ Vgl. INKAR (Indikatoren und Karten zur Raumentwicklung) des Bundesamtes für Bauwesen und Raumordnung.
} 
der Generation 50plus deutlich geringer ausfallen, als dies für heutige Senioren bekannt ist.

\subsection{Rein in die Stadt: Reurbanisierung und Umzug in die Kernstadt}

Nach der Analyse der Suburbanisierungstendenzen steht im Folgenden der ihr entgegengerichtete Prozess der Reurbanisierung im Mittelpunkt, der derzeit sowohl in der Tagespresse als auch in der Fachliteratur als „Renaissance der Städte" intensiv diskutiert wird (z. B. Herfert 2002; Brühl/ Echter/Frölich von Bodelschwingh et al. 2005; Große Starmann/Gebert/Schmidt 2006; Kuhn 2007), auch wenn aktuelle Wanderungsdaten noch keine Zuwanderung der Älteren in bedeutsamem Umfang erkennen lassen (Sturm/Meyer 2008: 243).

In einer Vielzahl der aktuellen Studien über Reurbanisierung wird das Phänomen qualitativ untersucht und von einer „Hinwendung zum Wohnen, Arbeiten und Leben in der Stadt“ sowie einer ,neuen Akzeptanz des Wohnens in der Innenstadt" gesprochen (Kuhn 2007: 125). Dabei erfolgt ein Rückbezug auf Untersuchungen zu Wanderungsmotiven. Allerdings wurden beispielsweise in der viel zitierten Studie des Deutschen Instituts für Urbanistik (Brühl/Echter/Frölich von Bodelschwingh et al. 2005) lediglich Personen befragt, die in ein innerstädtisches Quartier umgezogen sind. Gerade bei dieser Personengruppe kann die Akzeptanz des Wohnens in der Innenstadt jedoch vorausgesetzt werden. Wenn „das suburbane Lebensmodell tatsächlich in Frage gestellt" ist (Kuhn 2007: 126), dann müssten auch immer mehr „Suburbaniten“ dem Wohnen in der Stadt (wieder) aufgeschlossener gegenüberstehen. Hesse (2008: 416) macht Reurbanisierung an einer „Umkehr des längerfristigen Bedeutungsverlustes der Kernstädte im Rahmen der Suburbanisierung" fest, sieht den Prozess jedoch mehr als „Ausdruck und Resultat diskursiver Konstruktionen“ und weniger auf entsprechende Bevölkerungs- und Arbeitsplatzentwicklungen der Städte zurückführbar. In manchen Städten wie Leipzig wird Reurbanisierung ausdrücklich als „normatives Ziel der Stadterneuerung“ formuliert (Steinführer/Haase/Kabisch 2009: 186).

Auch die quantitativen Ansätze, das Phänomen zu erfassen, unterscheiden sich. Gans (2002: 160) versteht unter dem Prozess der Reurbanisierung „eine Phase der Stadtentwicklung, in der die Kernstadt eine relativ günstigere Bevölkerungsentwicklung als der suburbane Raum verzeichnet“. Nach dieser Definition würde Reurbanisierung auch Prozesse umfassen, in denen eine Kernstadt und ihr Umland Bevölkerungsverluste erleiden, diese jedoch in der Kernstadt geringer sind als im Umland. Demnach wäre Reurbanisierung auch in schrumpfenden Städten möglich. Zudem könnte die relativ günstigere Bevölkerungsentwicklung auch durch natürliches Bevölkerungswachstum, durch
Zuzug aus anderen Städten, durch Zuzug aus dem ländlichen Raum sowie aus dem Ausland zustande kommen (vgl. auch z. B. Sturm/Meyer 2008: 230).

Reurbanisierung in schrumpfenden Städten sowie durch natürliche Bevölkerungsentwicklung schließt Herfert (2002) aus und beschränkt die Wanderungsprozesse auf Zuzüge aus dem suburbanen Raum. Er sieht Reurbanisierungsprozesse nur als gegeben, wenn eine Stadt ,wieder (...) Wanderungsgewinne aus dem Umland und insgesamt eine (...) Bevölkerungszunahme" verzeichnet (Herfert 2002: 335). Dieser Prozess lässt sich z. B. in Leipzig auch auf die abnehmende Suburbanisierung zurückführen. Die Bevölkerungsentwicklungen resultieren im Wesentlichen aus einem Wandel bzw. Wegfall dominanter Wanderungsströme. Die StadtUmland-Wanderungen laufen im Saldo gegen Null bzw. es überwiegen die Umland-Stadt-Wanderungen. Vorerst sind es vorrangig junge Bevölkerungsgruppen, die die zentripetalen Wanderungsströme dominieren und für die Reurbanisierung verantwortlich sind bzw. dahingehend Pläne äußern (Gans/Schmitz-Veltin/West 2010: 56).

Eine Untersuchung der Bevölkerungsentwicklung von Mittelzentren in Baden-Württemberg (Brachat-Schwarz 2008) stellt eine Trendwende der Entwicklung seit der Jahrhundertwende fest, die sich darin ausdrückt, dass seit 2001 Wanderungssalden in den Zentren positiver sind als in den Umlandgemeinden. Diese Entwicklung geht jedoch aus Ausbildungs- und Arbeitsplatzgründen überwiegend auf Wanderungsgewinne der 15- bis unter 30-Jährigen zurück und kann für höhere Altersgruppen lediglich an einem Rückgang früherer Verluste festgemacht werden. Auch die Nähe zu altersgruppenspezifischen Infrastrukturangeboten ist offenbar für über 65-Jährige in Baden-Württemberg kein Grund für vermehrte Umzüge in die Zentren. Auch Untersuchungen in Aachen (Wörmer 2010) haben Zuzüge der Generation der 15- bis 30-Jährigen als Hauptursache für Bevölkerungszuwächse identifiziert. Die jungen Bildungsund Arbeitsplatzwanderer, die zum Teil aus ostdeutschen Regionen in westdeutsche Kernstädte wandern, stellen damit in vielen Zentren offenbar die wesentlichen Träger der aktuellen Reurbanisierungswelle dar (Sturm/Meyer 2008: 240). Sie könnte jedoch auch „das Ergebnis eines Struktureffektes sein, da sich diese stark besetzte Kohorte der ,DDR-Babyboomer ${ }^{68}$ gerade (noch) in der Phase der ersten Haushaltsgründung befindet" (Arbeitskreis Städte und Regionen der Deutschen Gesellschaft für Demographie 2007: 3).

Basierend auf Umfragen in ausgewählten Städten wird von einigen Autoren eine Reurbanisierung bzw. „Renaissance der Städte“ nur für Teile der zukünftigen Senio-

\footnotetext{
${ }^{8}$ Damit ist die Generation der Ende der 1970er bis Ende der 1980er Jahre Geborenen gemeint.
} 
rengeneration vorhergesagt (Rauterberg 20059; Große Starmann/Gebert/Schmidt 2006; Glasze/Graze 2007). ${ }^{10}$ In diesen Untersuchungen werden häufig ausschließlich „Suburbaniten" bezüglich ihrer Umzugsabsichten bzw. ihrer Umzugsbereitschaft in die Stadt befragt. Meist werden den ermittelten Werten nicht Befragungen von Stadtbewohnern bezüglich ihrer Umzugsabsichten in den suburbanen Raum gegenübergestellt. Somit kann auch nicht der Umfang der jeweiligen Wanderungsströme miteinander verglichen werden, wie dies bei der Auswertung von Bevölkerungs- und Wanderungsstatistiken möglich ist (z. B. bei Herfert 2002 und Sturm/Meyer 2008). Im Mainzer Umland planten die „Suburbaniten“ der Generation 50plus nur zu $5 \%$ konkret einen Umzug in eine Stadt. Von diesen $5 \%$ gaben $70 \%$ an, in die Stadt Mainz ziehen zu wollen (Glasze/Graze 2007: 472).

Die Auswertung der hier vorgestellten Befragungen in München, Aachen und Karlsruhe ergab unterschiedlich große, jedoch insgesamt sehr geringe Reurbanisierungstendenzen der Generation 50plus. Von den Karlsruher „Suburbaniten" hatte kein einziger künftig einen Umzug nach Karlsruhe geplant, in München waren es zwei (weniger als $1 \%$ der insgesamt 273 Befragten) und in Aachen sechs Personen (2\% der 295 Befragten). Weitere Analysen sind aufgrund der geringen Fallzahl nicht möglich.

Auch wenn in den Regionen Karlsruhe und Aachen derzeit weder aus unseren Daten noch aus den jeweiligen Wanderungsstatistiken Anzeichen für einen Reurbanisierungstrend der Generation 50plus erkennbar sind, wird nicht selten in Zukunft eine größere Zuwanderung von Senioren erwartet oder zumindest erhofft. Aus Sicht der Stadt Karlsruhe könnte dieser Trend aktuell am mangelnden Wohnungsangebot scheitern (Stadt Karlsruhe 2009: 55). Auch die Stadt Aachen konstatiert seit etwa zehn Jahren eine zunehmende Nachfrage von aktiven 60-jährigen Paaren nach attraktiven Wohnlagen (Stadt Aachen 2010: 7).

In den qualitativen Interviews unserer Untersuchung in den Münchner Umlandgemeinden äußerten insbesondere einige Befragte aus Vaterstetten, sich im Alter vorstellen zu

\footnotetext{
${ }^{9}$ Rauterberg (2005: 33) geht davon aus, dass es in Zukunft besonders ehemalige „Suburbaniten“ nach dem Auszug der Kinder aus dem „eintönigen Suburbia“ in die Städte ziehen wird. Vor allem denkt er dabei an die „Woopies“ (Well-off older people), ,die sich von ihrem Besitz lösen“ (gestützt auf eine Studie des Bochumer Instituts für Wohnungswesen, Immobilienwirtschaft, Stadt- und Regionalentwicklung (InWIS), nach der jeder dritte Deutsche über 50 das urbane Leben favorisiere)

${ }^{10}$ In einer Internet-Befragung der gut situierten Kunden der DB Immobilien (Plambeck 2006) zeigten sich klare Präferenzen hinsichtlich eines Alterswohnsitzes an einem stadtnahen, aber dennoch ruhigen Wohnstandort. Insbesondere unter denjenigen, die heute an einem suburbanen Standort wohnen, wird ein Umzug in ein urbaneres Umfeld in Betracht gezogen, an dem man leichter am städtischen Leben teilhaben kann.
}

können, nach München und dort sogar in die Innenstadt zu ziehen, entweder weil sie vor ihrem Umzug nach Vaterstetten in München wohnten oder ihnen Vaterstetten immer zu wenig urban war. Die Dachauer Interviewpartner äußerten derartige Absichten nicht.

\subsection{Persistenz}

Aus dem „Alterssurvey“ (Deutsches Zentrum für Altersfragen 1996) und anderen Studien (Friedrich 1995; Schneider-Sliwa 2004a; Schneider-Sliwa 2004b; Zaugg/van Wezemael/Odermatt 2004) ist bekannt, dass mehr als $80 \%$ der Menschen über 55 Jahre ihre Wohnsituation am liebsten unverändert lassen würden (vgl. auch Friedrich 2001a; Friedrich 2001b; Friedrich 2002). Auch vor dem Hintergrund des derzeit kontinuierlich absinkenden Binnenwanderungsvolumens erscheint es fraglich, ob es zu den prognostizierten Veränderungen kommen wird, besonders wenn man die sinkenden Binnenwanderungsraten der 65Jährigen und Älteren betrachtet (vgl. Friedrich 2009). Auch Hirschle/Schürt (2008) kommen bei einer Auswertung intraregionaler Wanderungsströme zu dem Schluss, „dass die Generation $50+$ doch weniger mobil ist bzw. seltener ihren Wohnstandort verlagert, als weithin angenommen" und dass zudem ,eine Änderung der Präferenz zum Wohnen in städtischen Lagen (...) nicht festzustellen“ ist (Hirschle/ Schürt 2008: 218). Man kann eher davon ausgehen, dass die zukünftigen Senioren dann, wenn sie es sich finanziell leisten und es gesundheitlich bewältigen können, versuchen werden, ihren geäußerten Absichten nach Persistenz nachzukommen.

Die Umzugsbereitschaft ist in hohem Maße von Faktoren wie dem Lebensstil (vgl. Herding 2006) oder auch der bisherigen Wohndauer und dem Wohneigentum sowie dessen Wiederverkaufswert (Glasze/Graze 2007: 472) abhängig. Anstelle eines Umzugs werden nicht selten beachtliche Investitionen in Umbauten, Renovierungen oder neue Einrichtungen geplant, so dass Verbesserungen der Wohnsituation bei einem Beibehalten der Wohnung und des Standorts möglich werden (Oswald/Hieber/Mollenkopf et al. 2003: 60 f.). Nach einer Studie der Landesbausparkassen zählt ein Drittel ihrer Kunden zu dieser Gruppe, ein weiteres Drittel gilt als „umzugsbereit“ (vorwiegend Akademiker) und der Rest wünscht keine wohnliche Veränderung (LBS 2006: 25).

In der Befragung in der Region München spielte die Bewertung der aktuellen Wohnstandorte für die zukünftige Planung sowohl in der qualitativen als auch in der quantitativen Erhebung eine große Rolle. Durch fast alle qualitativen Interviews zog sich als roter Faden, dass für die meisten Befragten ihre jeweilige Wohngemeinde der ideale Wohnort ist und das derzeitige Wohnviertel deutlich mehr Vorteile als Nachteile besitzt. 
In allen drei Städten äußerten sich die Befragten der quantitativen Erhebung zu mehr als $80 \%$ zufrieden oder sehr zufrieden mit der Anbindung an den öffentlichen Nahverkehr, dem Angebot an Grün- und Freiflächen sowie der Sicherheit. ${ }^{11}$ In München wird im Vergleich zu den anderen beiden Städten insbesondere das Angebot an Einkaufsmöglichkeiten geschätzt (von $83 \%$ der Befragten). Karlsruher sind besonders zufrieden mit den Sportmöglichkeiten ( $82 \%$ der Befragten) und Aachener mit dem Angebot an Ärzten bzw. der medizinischen Versorgung (95\% der Befragten) sowie dem Kulturangebot ( $83 \%$ der Befragten). Wie zu erwarten, werden diese Aspekte in den jeweiligen Umlandgemeinden weniger positiv gesehen, wohingegen z. B. Ruhe oder Parkmöglichkeiten dort besser bewertet werden. Die Befragten reflektieren die Vor- und Nachteile ihrer jeweiligen Standorte durchaus kritisch. Dennoch zeichnet sich auch in den quantitativen Erhebungen ab, dass die Generation 50plus offenbar in Stadtteilen wohnt, die in hohem Maße ihren Präferenzen entsprechen.

Auf die Frage nach möglichen Veränderungen der Wohnsituation antworteten in München $42 \%$ aller Befragten, dass für sie grundsätzlich keine Veränderung in Frage käme bzw. dass sie lediglich innerhalb des Stadtteils, in dem sie derzeit wohnen, einen Umzug geplant haben. Auch in Aachen (41\%) und Karlsruhe (44\%) bevorzugt ein Großteil der Befragten, die aktuelle Wohnsituation auch nach dem Eintritt in den Ruhestand beizubehalten. In den suburbanen Gemeinden sind die Werte noch höher und erreichen bis zu 50 \% (im Umland Karlsruhe: 50 \%, im Umland München: $49 \%$, im Umland Aachen: $48 \%$ ).

Vor allem die geringe Zahl derer, die bereits konkrete Pläne für einen Umzug nach dem Ruhestand hat, stützt die Persistenz-These. In den qualitativen Interviews äußerten die meisten Befragten, nicht nur weiterhin in München, sondern auch in ihrem jeweiligen Stadtteil und am besten auch in der jetzigen Wohnung bleiben zu wollen: „Aber ich würde jetzt nie mehr wegziehen, weil die Wohnqualität hier ist enorm, weil es keine Hauptverkehrsstraße ist, die Kaiserstraße, Schlafzimmer hinten naus geht, ist man so ruhig. (...) Also den Standort München aufgeben - nein, da müsste mein Mann schon alleine gehen. "12 Insbesondere von den Innenstadtbewohnern denken nur wenige darüber nach, an den Stadtrand oder in eine Umlandgemeinde zu ziehen. Auch die Bewohner der am Rande gelegenen Stadtbezirke und der Umlandgemeinden haben den Wunsch, am aktuellen Wohnstandort zu bleiben, da sie entweder im eigenen Haus oder in einem ruhigen und grünen Umfeld wohnen,

\footnotetext{
${ }^{11}$ Die Befragungen fanden vor den Gewalttaten in den Münchener Sund U-Bahn-Stationen statt. Eine Befragung zum jetzigen Zeitpunkt könnte durchaus zu anderen Ergebnissen führen.

12 59-jährige technische Zeichnerin, arbeitslos, München-Schwabing.
}

das genau ihren Präferenzen entspricht, und sie intensive soziale und familiäre Netze vor Ort besitzen.

Diese Ausführungen korrespondieren mit den Ergebnissen der quantitativen Befragung. Die Befragten im Umland Karlsruhes äußerten am häufigsten die Absicht, am Wohnstandort zu verbleiben und weisen zugleich mit $81 \%$ die höchste Wohneigentumsquote auf. Im Umland Münchens und Aachens leben $71 \%$ bzw. $69 \%$ der Befragten im Eigentum. In den Städten wurden erwartungsgemäß geringere Werte erreicht, wobei die Befragten in den Städten Karlsruhe und Aachen (59\% bzw. $57 \%$ ) überdurchschnittlich häufig im eigenen Haus oder der eigenen Wohnung leben, während dies in der Stadt München nur auf 29 \% der Befragten zutrifft.

Die außerordentlich hohe Zufriedenheit mit dem aktuellen Wohnort, die in den qualitativen Interviews zum Ausdruck gekommene große Identifikation mit dem aktuellen Wohnstandort und dem Wohnumfeld sowie die zum Teil hohen Eigentumsquoten lassen weiterhin eine große Persistenz der zukünftigen Senioren erwarten.

\section{Ergebnis: mehr Persistenz als Trendwende}

Im Vergleich zur aktuellen negativen Wanderungsbilanz der heutigen Senioren in Kernstädten (Bucher/Heins 2001; Referat für Stadtplanung und Bauordnung München 2002), die für fast alle deutschen Großstädte gilt, kann in allen drei Untersuchungsstädten für die Zukunft nicht mit einer weiteren intensiven Abwanderung der kommenden älteren Generation in den suburbanen Raum gerechnet werden. Aber auch aus den suburbanen Gemeinden ist nicht mit vermehrten Umzügen in die Stadt zu rechnen. Grundsätzlich scheinen sich die Präferenzen, die bei der vorangegangenen Wohnstandortwahl bestanden, nicht so stark geändert zu haben, dass nun im höheren Alter oder nach dem Eintritt in den Ruhestand ein Wohnen in einer gänzlich anderen Umgebung gewünscht wird. Zudem müssen Anpassungen bzw. die Gewöhnung an einen Standort und an ein räumliches und soziales Umfeld bei der Bewertung mit in Betracht gezogen werden.

Bei allen diskutierten Migrationsabsichten wie auch bei dem Wunsch der Persistenz spielen vor allem in München finanzielle Erwägungen eine wesentliche Rolle. Sowohl der Umzug in das suburbane Umland, in eine andere deutsche Stadt als auch in ein anderes Land wurden in der bayerischen Landeshauptstadt überwiegend aus Kostengründen erwogen. Die Möglichkeit, am derzeitigen Wohnstandort wohnen bleiben zu können, wird dort am ehesten aufgrund der künftigen finanziellen Situation in Frage gestellt. Die eigenen finanziellen Ressourcen (als Ressourcen und MikroStrukturen im Sinne von Giddens) und die Kostenentwicklung des urbanen und suburbanen Wohnungsmarktes (als 


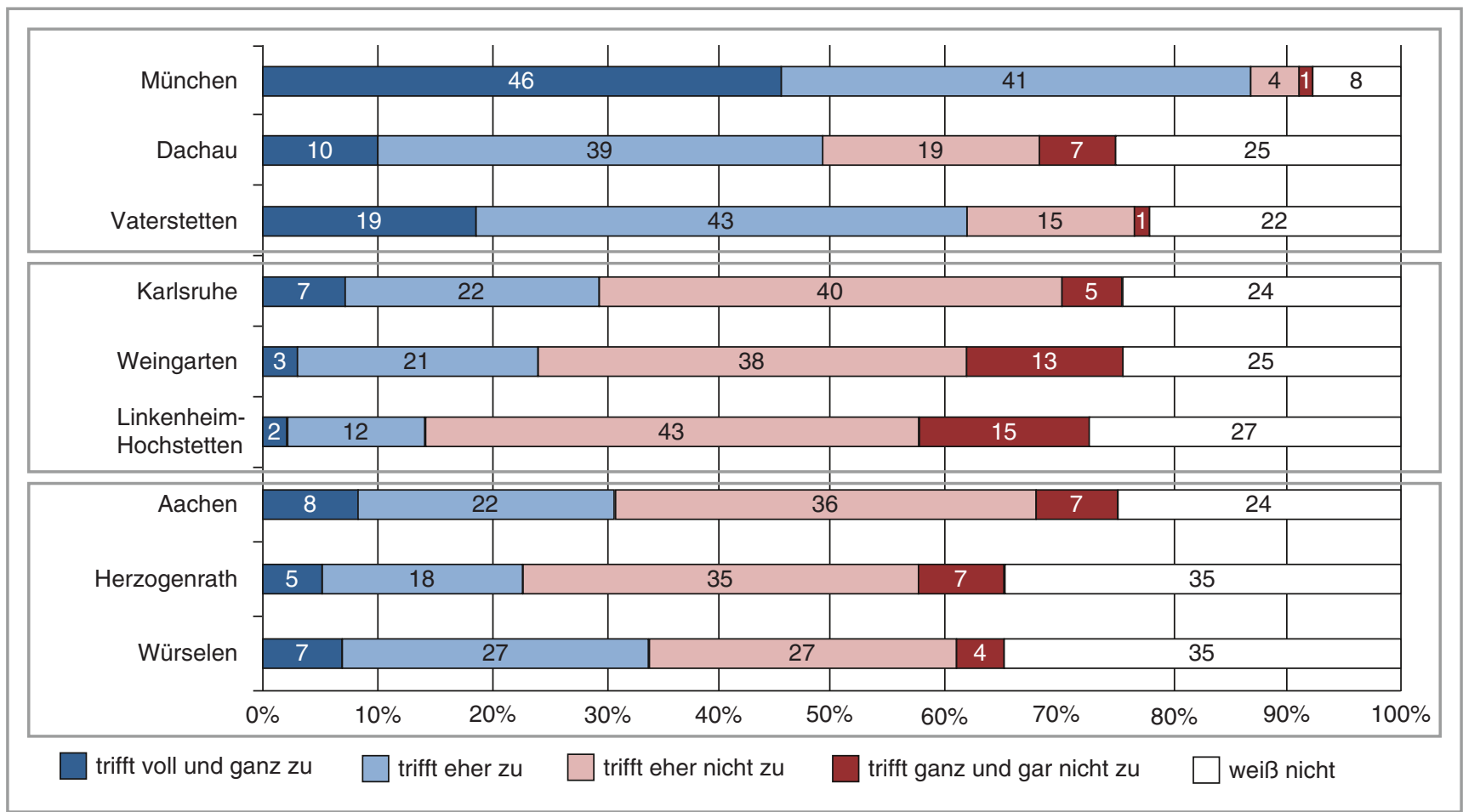

Abb. 1 „Dieser Ort ist zu teuer für Seniorinnen und Senioren“

Makro-Strukturen) wirken damit als beachtliche constraints im Sinne von Hägerstrand.

Die größten Sorgen bereiten den Befragten in München die mit dem Eintritt in den Ruhestand verbundenen Einkommensreduzierungen. Insbesondere die Bezieher niedriger Einkommen und Selbstständige mit bislang ungenügender Altersvorsorge äußerten dahingehend Zukunftsängste. Ein 52-jähriger Sachbearbeiter aus München-Obergiesing fasst den geringen Spielraum seiner künftigen Wohnungs- und Wohnortwahl wie folgt zusammen: „Man wird froh sein, wenn man das Geld für die Miete zusammenkriegt.“ Aber auch in den gentrifizierten Stadtbezirken Schwabing und Isarvorstadt werden die Mieten als überhöht empfunden und die Befragten berichten von finanziell motivierten Fortzügen von Freunden: „Mittlerweile heißt es ,Ich kann die Mieten nicht mehr bezahlen', und ich ziehe nach Berlin. Das hat auch eine ganze Reihe, auch leider von meinen Freunden gemacht, aber das würde ich nicht tun."13

Deutlich anders ist die Situation in Aachen und Karlsruhe. Hier bereiten die eher moderaten Mietkosten den Befragten weniger Sorgen, sich die derzeitige Wohnung im Alter nicht mehr leisten zu können. Zudem wird ein Verbleiben am urbanen Wohnstandort durch die höhere Eigentumsquote befördert. Die Lebenskonzepte der Befragten werden somit in hohem Maße mit dem räumlichen Kontext (hier

\footnotetext{
${ }^{13}$ 59-jähriger selbstständiger Schreiner, München-Schwabing.
}

dem lokalen Wohnungsmarkt) in Bezug gesetzt und vor diesem Hintergrund entwickelt.

In aller Deutlichkeit zeigten sich Unterschiede des Preisniveaus bei der Zustimmung bzw. Ablehnung der Aussage, die jeweilige Stadt sei „zu teuer für Seniorinnen und Senioren“. In der Stadt München stimmten dem $46 \%$ voll und ganz zu und weitere $41 \%$ stimmten dem eher zu. Erwartungsgemäß betrifft dies auch die Umlandgemeinden Münchens, wenn auch nicht in solch gravierendem Ausmaß. In Aachen und Karlsruhe stimmten dagegen nur $8 \%$ bzw. $7 \%$ der Befragten der Aussage voll und ganz zu, ihre Stadt sei zu teuer für Seniorinnen und Senioren, fast die Hälfte der Befragten lehnte diese Aussage ab. In den Umlandgemeinden Karlsruhes stellt das Preisniveau für die Befragten offenbar das geringste Problem dar (vgl. Abb. 1).

Die Befragten in den Großstadtregionen München, Karlsruhe und Aachen planen weder in größerem Ausmaß, ihre Städte im Alter zu verlassen, noch zieht es die ehemaligen „Suburbaniten“ in großer Zahl in die Städte. Das Phantom des rüstigen „Reurbaniten“ geistert durch mancherlei Diskurse und mag durchaus dem heimlich gehegten Wunsch mancher Stadtpolitiker und Stadtforscher entsprechen. In Städte zurückkehrende „Suburbaniten“ sind in Einzelfällen durchaus zu beobachten, sie treten jedoch weder in Wanderungsstatistiken noch in empirischen Studien wie der vorliegenden in größerer Zahl in Erscheinung. Wenn man eine Renaissance der Städte jedoch daran festmacht, dass 
Städte heute weniger oft als früher verlassen werden, dann sind auch für die Generation 50plus Reurbanisierungstendenzen feststellbar. Die jungen Alten sind somit keine ,neuen“ „Urbaniten“, sie wollen nur ,weiterhin“ „Urbaniten" bleiben.

\section{Literatur}

Amrhein, L. (2004): Die Bedeutung von Situations- und Handlungsmodellen für das Leben im Alter. In: Blüher, S.; Stosberg, M (Hrsg.): Neue Vergesellschaftungsformen des Alter(n)s. Wiesbaden, 53-86. = Alter(n) und Gesellschaft, Band 9.

Arbeitskreis Städte und Regionen der Deutschen Gesellschaft für Demographie e.V. (2007): Wesentliche Strukturen und Trends des demographischen Wandels in den Städten Deutschlands - Diskussionsgrundlage. Autorenpapier zur Dezembertagung des Arbeitskreises mit dem Bundesamt für Bauwesen und Raumordnung am 6. und 7. Dezember 2007 in Berlin.

Beck, U. (1986): Risikogesellschaft. Auf dem Weg in eine andere Moderne. Frankfurt am Main.

Boerner, S.; Schramm, F. (1998): Fluktuationsneigung in den neunziger Jahren: Eine empirische Analyse anhand des Sozio-oekonomischen Panels. In: Zeitschrift für Personalforschung 98, 1, 79-97.

Brachat-Schwarz, W. (2008): Reurbanisierung - Gibt es eine „Renaissance der Städte" in Baden-Württemberg? In: Statistisches Monatsheft Baden-Württemberg 11, 5-13.

Breuer, T. (2003): Deutsche Rentnerresidenten auf den Kanarischen Inseln: In: Geographische Rundschau 55, 5, 44-51.

Breuer, T. (2004): Successful Aging auf den Kanarischen Inseln? Versuch einer Typologie von Alterns-Strategien deutscher Altersmigranten. In: Europa Regional 12, 3, 122-131.

Breuer, T. (2005): Retirement Migration or rather Second-Home Tourism? German Senior Citizens on the Canary Islands. Dedicated to Jürgen Bähr on his 65th birthday. In: Die Erde 136, 3, 313-333.

Breuer, T.; Müller, D. (2005): Grenzenlose Mobilität? Deutsche Auslandsbevölkerung in Europa. In: Leibniz-Institut für Länderkunde (Hrsg.): Nationalatlas Bundesrepublik Deutschland, Band 11: Deutschland in der Welt. München, 68-71.

Brühl, H.; Echter, C.-P.; Frölich von Bodelschwingh, F. et al. (2005): Wohnen in der Innenstadt. Eine Renaissance? Berlin. = Difu-Beiträge zur Stadtforschung, Nr. 41.

Bucher, H.; Heins, F. (2001): Altersselektivität der Wanderungen. In: Institut für Länderkunde Leipzig (Hrsg.): Nationalatlas Bundesrepublik Deutschland, Band 4: Bevölkerung. Berlin/Heidelberg, 120-123.

Buck, C. (2005): Zweit- und Alterswohnsitze von Deutschen an der Costa Blanca. Räumliche Identifikation und soziale Netzwerke im höheren Erwachsenenalter am Beispiel der Gemeinde Els Poblets. Dissertation am Fachbereich Geowissenschaften der Martin-Luther-Universität Halle-Wittenberg.

Deutsches Zentrum für Altersfragen (1996): Der Alterssurvey. Berlin.

Eichener, V. (2001): Mobil bleiben! Über das Umziehen im Alter. In: Schader-Stiftung (Hrsg.): wohn:wandel. Szenarien, Prognosen, Optionen zur Zukunft des Wohnens. Darmstadt, 174-185.

Flöthmann, E.-J. (1997): Der biographische Ansatz in der Binnenwanderungsforschung. In: IMIS-Beiträge 5, 25-45.

Friedrich, K. (1995): Altern in räumlicher Umwelt. Sozialräumliche Interaktionsmuster älterer Menschen in Deutschland und den USA. Darmstadt.

Friedrich, K. (2001a): Binnenwanderung älterer Menschen. In: Institut für Länderkunde Leipzig (Hrsg.): Nationalatlas Bundesrepublik Deutschland, Band 4: Bevölkerung. Berlin/Heidelberg, 124-125.
Friedrich, K. (2001b): Altengerechte Wohnumgebungen. In: Flade, A.; Limbourg, M.; Schlag, B (Hrsg.): Mobilität älterer Menschen. Opladen, 155-168.

Friedrich, K. (2002): Migrationen im Alter. In: Schlag, B.; Megel, K. (Hrsg.): Mobilität und gesellschaftliche Partizipation im Alter. Stuttgart, 87-96. $=$ Schriftenreihe des Bundesministeriums für Familie, Senioren, Frauen und Jugend, Nr. 230.

Friedrich, K. (2009): Wohnen im Alter. Fluidität und Konstanz der Anspruchsmuster in raumzeitlicher Perspektive. In: Blättel-Mink, B.; Kramer, C (Hrsg.): Doing Aging - Weibliche Perspektiven des Älterwerdens. Baden-Baden, 45-54. = Schriften des Heidelberger Instituts für Interdisziplinäre Frauen- und Geschlechterforschung (HIFI) e.V., Nr. 7.

Friedrich, K.; Kaiser, C. (2001): Rentnersiedlungen auf Mallorca? In: Europa Regional 9, 4, 204-211.

Gans, P. (2002): Reurbanisierung. In: Brunotte, E.; Gebhardt, H.; Meurer, M (Hrsg.): Lexikon der Geographie, Band 3. Heidelberg, 160.

Gans, P.; Schmitz-Veltin, A.; West, C. (2010): Wohnstandortentscheidungen von Haushalten am Beispiel Mannheim. In: Raumforschung und Raumordnung 68, 1, 49-59.

Giddens, A. (1988): Die „Theorie der Strukturierung“. Ein Interview von Bernd Kießling mit Anthony Giddens. In: Zeitschrift für Soziologie 17, 286-295.

Giddens, A. (1997): Die Konstitution der Gesellschaft. Grundzüge einer Theorie der Strukturierung. Frankfurt am Main.

Glasze, G.; Graze, P. (2007): Raus aus Suburbia - rein in die Stadt? Studie zur zukünftigen Wohnmobilität von Suburbaniten der Generation $50+$. In: Raumforschung und Raumordnung 65, 5, 467-473.

Große Starmann, C.; Gebert, J.; Schmidt, K. (2006): Mittendrin und nicht am Rand. Online unter: http://wegweiser-kommune.de/ themenkonzepte/demographie/download/pdf/Urbanezentren.pdf (letzter Zugriff am 11.05.2010)

Hägerstrand, T. (1975): Space, Time and Human Conditions. In: Karlqvist, A.; Snickars, F (Hrsg.): Dynamic Allocation of Urban Space. Farnborough, 3-12.

Heinze, R. G.; Eichener, V.; Naegele, G. et al. (1997): Neue Wohnung auch im Alter. Folgerungen aus dem demographischen Wandel für Wohnungspolitik und Wohnungswirtschaft. Online unter: http://www.schader-stiftung.de/docs/neue_wohnung_kurzfassung_ok.pdf (letzter Zugriff am 11.05.2010).

Herding, M. (2006): Lebensstile und räumliche Mobilität von Menschen im Dritten Lebensalter - Eine empirische Untersuchung am Beispiel zweier Wohngebiete in Münster. Diplomarbeit am Geographischen Institut der Westfälischen Wilhelms-Universität Münster.

Herfert, G. (2002): Disurbanisierung und Reurbanisierung. Polarisierte Raumentwicklung in der ostdeutschen Schrumpfungslandschaft. In: Raumforschung und Raumordnung 60, 5-6, 334-344.

Herfert, G.; Schulz, M. (2002): Wohnsuburbanisierung in Verdichtungsräumen. In: Institut für Länderkunde Leipzig (Hrsg.): Nationalatlas Bundesrepublik Deutschland, Band 5: Dörfer und Städte. Berlin/Heidelberg, 124-127.

Hesse, M. (2008): Reurbanisierung? Urbane Diskurse, Deutungskonkurrenzen, konzeptionelle Konfusion. In: Raumforschung und Raumordnung 66, 5, 415-428.

Hirschle, M.; Schürt, A. (2008): Suburbanisierung ... und kein Ende in Sicht? Intraregionale Wanderungen und Wohnungsmärkte. In: Informationen zur Raumentwicklung 3/4, 211-227.

Inglehart, R. (1998): Modernisierung und Postmodernisierung. Frankfurt am Main.

Kramer, C.; Pfaffenbach, C. (2007): Alt werden und jung bleiben - Die Region München als Lebensmittelpunkt zukünftiger Senioren? In: Raumforschung und Raumordnung 65, 5, 393-406. 
Kramer, C.; Pfaffenbach, C. (2009): Persistence Preferred - on Future Residential (Im)Mobility among the Generation 50plus. In: Erdkunde 63, 2, 161-172.

Kuhn, G. (2007): Reurbanisierung, Renaissance der Städte und Stadtwohnen. In: Informationen zur modernen Stadtgeschichte 2, 121-130.

LBS (Bundesgeschäftsstelle Landesbausparkassen im Deutschen Sparkassen- und Giroverband) (2006): Die Generationen über 50. Wohnsituation, Potenziale und Perspektiven. Online unter: http://www.lbs.de/lbs/pics/upload/tfmedia1/HBMAAlnaWMg. pdf (letzter Zugriff am 11.05.2010).

Meusburger, P. (1999): Subjekt - Organisation - Region. Fragen an die subjektzentrierte Handlungstheorie. In: Meusburger, P. (Hrsg.): Handlungszentrierte Sozialgeographie. Stuttgart, 95-132. = Erdkundliches Wissen, Nr. 130.

Oswald, F.; Hieber, A.; Mollenkopf, H. et al. (2003): Heidelberg 2002: Wohnwünsche und Wohnwirklichkeiten: Abschlussbericht. Heidelberg. $=$ Forschungsberichte aus dem Deutschen Zentrum für Alternsforschung, Nr. 15.

Pfaffenbach, C. (2005): Die Transformation des Lebens - biographischgeographische Forschungen in Ostdeutschland. In: Berichte zur deutschen Landeskunde 76, 1, 59-76.

Plambeck, K. (2006): „Quo vadis Papa?“ - wo wohnen im Alter. Diplomarbeit am Geographischen Institut der Ruprecht-Karls-Universität Heidelberg.

Rauterberg, H. (2005): Neue Heimat Stadt. In: Die Zeit, Nr. 34, 18.08.2005, 33 .

Referat für Stadtplanung und Bauordnung der Landeshauptstadt München (2002): Raus aus der Stadt? Untersuchung der Motive von Fortzügen aus München in das Umland 1998-2000. München, unveröffentlichtes Manuskript.

Referat für Stadtplanung und Bauordnung der Landeshauptstadt München (2004): Folgen des soziodemografischen Wandels und Herausforderungen für die Stadtentwicklung. München, unveröffentlichtes Manuskript.
Schneider-Sliwa, R. (2004a): Städtische Umwelt im Alter. Präferenzen älterer Menschen zu Wohnumfeld- und Quartiersgestaltung. Basel. $=$ Basler Stadt- und Regionalforschung, Band 26.

Schneider-Sliwa, R. (2004b): Städtische Umwelt im Alter. In: Geographica Helvetica 59, 4, 300-312.

Stadt Aachen (2010): Zurück in die Stadt? - Die neue Attraktivität der Städte. Fallstudie Aachen. BBSR-Ressortforschungsbericht. Aachen, unveröffentlichtes Manuskript.

Stadt Karlsruhe, Amt für Stadtentwicklung (2009): Demographischer Wandel in Karlsruhe. Zu- und Fortzüge über die Stadtgrenzen 1999-2008. Karlsruhe. = Beiträge zur Stadtentwicklung, Nr. 26.

Steinführer, A.; Haase, A.; Kabisch, S. (2009): Leipzig - Reurbanisierungsprozesse zwischen Planung und Realität. In: Kühn, M.; Liebmann, H (Hrsg.): Regenerierung der Städte. Strategien der Politik und Planung im Schrumpfungskontext. Wiesbaden, 176-194.

Sturm, G.; Meyer, K. (2008): „Hin und her“ oder „hin und weg“ - zur Ausdifferenzierung großstädtischer Wohnsuburbanisierung. In: Informationen zur Raumentwicklung 3/4, 229-243.

Wagner, M. (1989): Räumliche Mobilität im Lebensverlauf. Stuttgart.

Weichhart, P. (2003): Gesellschaftlicher Metabolismus und Action Settings. Die Verknüpfung von Sach- und Sozialstrukturen im alltagsweltlichen Handeln. In: Meusburger, P.; Schwan, T (Hrsg.): Humanökologie. Ansätze zur Überwindung der Natur-KulturDichotomie. Stuttgart, 15-44. = Erdkundliches Wissen, Nr. 135.

Weichhart, P. (2008): Entwicklungslinien der Sozialgeographie. Stuttgart. $=$ Sozialgeographie kompakt, Nr. 1 .

Werlen, B. (2007): Sozialgeographie. In: Gebhardt, H.; Glaser, R.; Radtke, U et al. (Hrsg.): Geographie. Physische Geographie und Humangeographie. München, 579-598.

Wörmer, S. (2010): Die Wiederentdeckung des städtischen Wohnens. Untersuchungen der Wanderungen in die Stadt Aachen im Kontext von Reurbanisierungsprozessen. München.

Zaugg, V.; van Wezemael, J. E.; Odermatt, A. (2004): Wohnraumversorgung für alte Menschen: ein Markt? In: Geographica Helvetica 59, 4, 313-321. Autorinnen: 